\title{
Histologic differential diagnosis of focal liver lesions by ultrasonically guided fine needle biopsy
}

\author{
B LIMBERG, W W HÖPKER, AND B KOMMERELL \\ From the Department of Internal Medicine, Department of Pathology, University of Heidelberg, Heidelberg, \\ FRG
}

SUMMARY Ultrasonically guided fine needle biopsies were carried out in 84 patients with focal liver lesions and were subsequently evaluated histologically. A correct diagnosis of malignancy was made in 46 of 52 patients with proven hepatic malignancy, showing an overall accuracy of $93 \%$. The specificity of the procedure was $100 \%$. Such histological examination not only enables the differentiation between primary and secondary hepatic malignancy; it has the additional advantage of making a more precise tumour description and at the same time it pinpoints the primary site of the tumour.

Imaging techniques such as dynamic ultrasound or computed tomography provide high resolution sectional images of focal liver lesions. It is not possible with a clinically acceptable degree of accuracy to distinguish between benign and malignant lesions with any of these imaging modalities, for an exact diagnosis a cytological or histological examination is necessary. Fine needle biopsy of the liver has been shown, to be a safe and accurate method for the cytological diagnosis of cancer; the diagnostic yield has been reported to be from $84-95 \%$. . $^{-5}$ The differentiation between primary and secondary hepatic malignancy, however, requires histological examination. Moreover, diagnosis of hepatocellular carcinoma cannot easily be accomplished by cytological examination alone, because regenerating hepatocytes - for example, in hepatitis or active cirrhosis, may show changes mimicking hepatocellular carcinoma."

On fine needle histological sampling tissue fragments are obtained, making possible a histological diagnosis with a more precise tumour description. The purpose of our study was to examine the differential diagnostic value of ultrasonically guided fine needle aspiration combined with histological

Address for correspondence: PD Dr Bernd Limberg. Medizinische Universitätsklinik, Bergheimerstr 58, D-6900 Heidelberg. Federal Republic of Germany.

Received for publication 1.3 June 1986. examination of the aspirated material in patients with suspected hepatic malignancy and focal hepatic lesions.

\section{Methods}

\section{PATIENTS}

Ultrasonically guided fine needle biopsies were carried out on 84 patients (average age: 59 years). Biopsies were done for the following indications: to verify the presence of liver metastases in patients with known malignant disease in order to determine the correct stage of the disease, to determine the nature of the primary tumour in patients with suspected hepatic malignancy and occult malignant disease, and to differentiate between malign and benign focal lesions with equivocal focal echo patterns.

Biopsies were obtained using a commercially available needle with an outer diameter of $0.9 \mathrm{~mm}(20$ gauge; Braun Melsungen; TSK-Supra). The ultrasound guided biopsies were done freehand. A $20 \mathrm{cc}$ syringe containing $0.9 \% \mathrm{NaCl}$ was attached to the needle. After local anaesthesia, the needle was introduced close to the transducer and visualised as it entered the sound field and was then introduced into the focal hepatic lesion. The needle was quickly moved back and forth several times during maximal aspiration. Three or four passes were made in each patient. The diameter of the focal hepatic lesions 
varied from 1.5 to $13 \mathrm{~cm}$ (mean: $3.9 \mathrm{~cm}$ ). In cystic lesions the fluid was aspirated and a biopsy was taken from the tissue next to the cyst. Material suitable for histological examination was obtained in 78 of 84 patients. The tissue fragments were fixed in $5 \%$ unbuffered formalin, embedded in paraplast and were stained by the Masson-Goldner, periodic acidSchiff (PAS) and the haematoxylin-eosin technique.

The diagnosis of malignant liver disease was considered proven when confirmatory tissue was obtained by surgery or at necropsy, or when the clinical course of the disease showed a malignancy. The diagnosis of benign focal liver lesion was considered correct when additional biochemical, radiologic, and endoscopic methods as well as the sonographic follow up and the clinical course of the disease over two years excluded a malignant disease of the liver. The diagnosis of haemangioma was confirmed by angio-computed tomography and in two cases by surgery. In patients with primary carcinoma of the liver alpha-1-fetoprotein (AFP) serum concentration was determined routinely and in some cases a laparoscopy combined with a direct large bore biopsy was undertaken.

\section{Results}

Fifty two of 84 patients who had undergone fine needle biopsy had hepatic malignancy confirmed by other invasive procedures, and imaging methods, as well as by the clinical course of the disease (Table 1). Fine needle biopsy results definitely proved to be positive for malignant disease in 46 of the 52 patients. There were six false negative results, but there were no false positive results; the overall accuracy was $93 \%$, and the specificity $100 \%$ (Table 2). Histological examination gave a more precise tumour description and with the exception of 10 cases a determination of the primary site of the tumour was possible (Figure).

In the current series where 15 patients have diagnostically proved hepatocellular carcinoma, raised serum AFP concentrations were the first clue to diagnosis in only $46 \%$ of the cases. In addition to fine needle biopsies, laparoscopies and large bore biopsies were carried out in six cases, but in these cases neither the laparoscopies nor the biopsie confirmed the diagnosis. In 13 out of these 15 patients with proved hepatocellular carcinoma histological examination of the ultrasonically guided fine needle biopsies proved to be positive; in one patient the biopsy was classified as negative, and in the other as adenoma (Table 3).

Thirty two of the 84 patients had a benign focal hepatic lesion (Table 4). In nine patients the diagnosis of a hepatic cyst was confirmed by aspiration of clear fluid; the biopsy taken from the neighbouring tissue showed normal liver parenchyma. In seven cases initial minimal aspiration was followed by an immediate spurt of blood, at which time the procedure was stopped and no further passes made into the lesion. In only one of these seven cases could a specimen for histological examination be taken. Aspiration of blood as well as the characteristic results obtained from computed tomography provided suggestive evidence of the presence of haemangioma. In nine patients biopsy specimens of echogenic lesions were reported by the pathologist as consistent with fatty infiltration of the liver and no malignant cells were encountered. In these patients other clinical and laboratory investigations and the follow up revealed no clinical signs of malignant disease. In two cases a resolving hepatitis was diagnosed and the subsequent sonographic changes over a period of months confirmed the diagnosis.

\section{Discussion}

Fine needle biopsy followed by cytologic examination has been proved to be a reliable method for the diagnosis of hepatic malignancy. The diagnostic yield has been reported to be from $84-95 \%$. ${ }^{1-5}$ Nonetheless the results of cytological examination are marred by the presence of false positives. ${ }^{78}$ Moreover, the differentiation between primary and secondary hepatic malignancy is difficult by cytological examination alone. ${ }^{9}$ In establishing a management plan for

Table 1 Sites of primary neoplasms in patients with malignant focal lesions

\begin{tabular}{lc}
\hline Primary tumour & Patients $(\boldsymbol{n})$ \\
\hline Liver & 15 \\
Stomach & 4 \\
Colon & 12 \\
Breast & 4 \\
Bronchogenic & 4 \\
Melanoma & 2 \\
Hodgkin's & 1 \\
Urinary bladder & 1 \\
Pancreas & 2 \\
Gall bladder & 1 \\
Unknown primary & 6 \\
Total & 52 \\
\hline
\end{tabular}

Table 2 Results of histologic examination of ultrasonically guided fine-needle biopsies of focal liver lesions

\begin{tabular}{lllll}
\hline $\begin{array}{l}\text { Malignant lesions } \\
\text { True }\end{array}$ & & & False \\
\cline { 1 - 1 } \cline { 4 - 5 } Positive & Negative & & Positive & Negative \\
$46 / 84$ & $32 / 84$ & & $0 / 84$ & $6 / 84$ \\
\hline
\end{tabular}

Overall accuracy: $93 \%$; specificity: $100 \%$. 

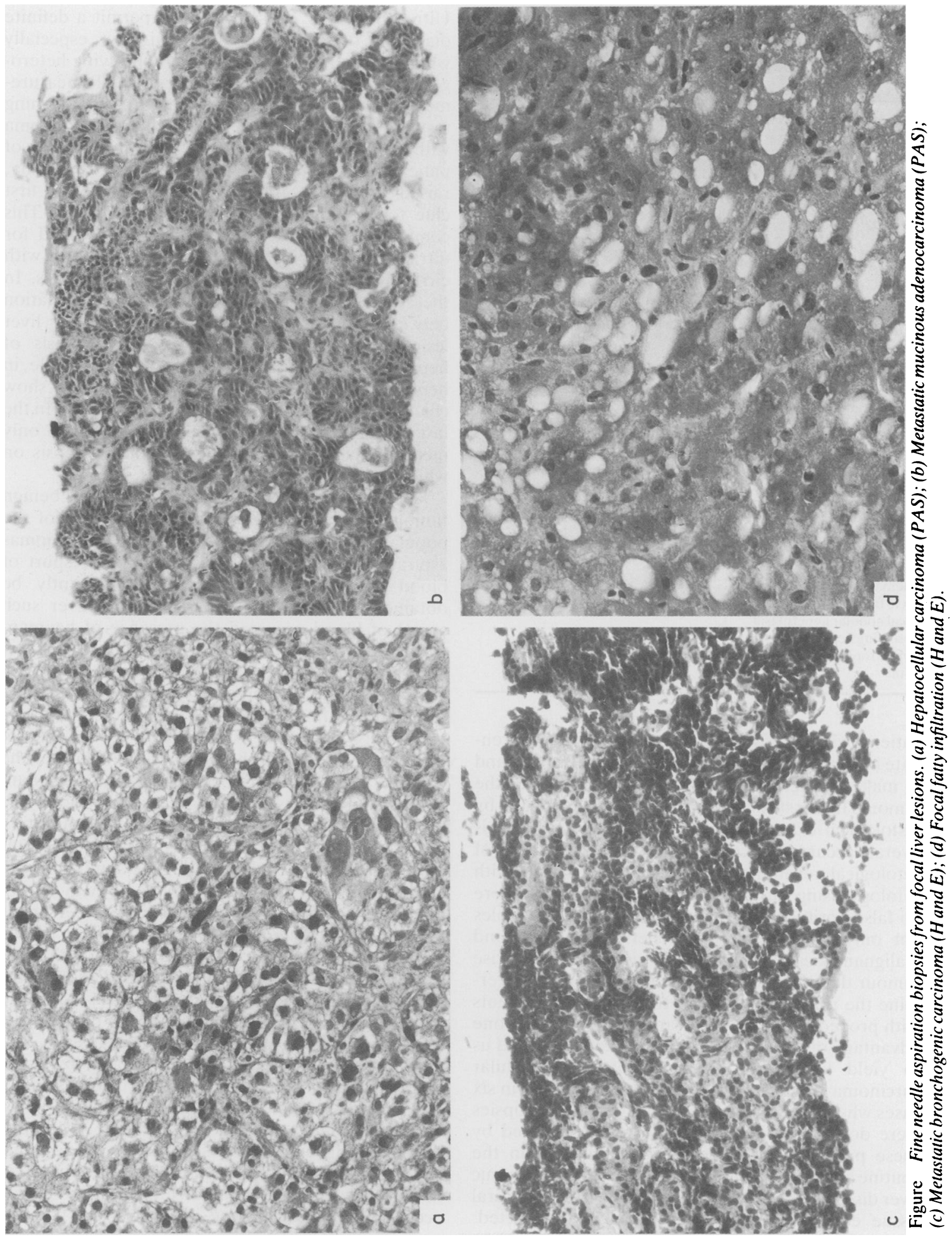
Table 3 Results of fine needle biopsy, laparoscopy and determination of serum Alpha-fetoprotein (AFP) concentrations in patients with proven hepatocellular carcinoma $(H C C)$

\begin{tabular}{cllc}
\hline & Fine needle biopsy & Laparoscopy/biopsy & AFP(ng/ml) \\
\hline 1 & HCC & Cirrhosis & 2500 \\
2 & HCC & Cirrhosis & 12 \\
3 & HCC & & 50 \\
4 & HCC & & 1100 \\
5 & HCC & Cirrhosis & 1050 \\
6 & Cirrhosis & & 1620 \\
7 & HCC & Chronic active hepatitis & 10 \\
8 & HCC & & 9 \\
9 & HCC & & 12 \\
10 & HCC & & 31 \\
11 & HCC & Chronic active hepatitis & 42 \\
12 & Adenoma & & 19 \\
13 & HCC & Cirrhosis & 3500 \\
14 & HCC & & 850 \\
15 & HCC & & 980 \\
Positive & $13 / 15$ & $0 / 6$ & $7 / 15$ \\
\hline
\end{tabular}

Table 4 Benign focal hepatic lesions

\begin{tabular}{lc}
\hline & Patients $(n)$ \\
\hline Cyst & 9 \\
Abscess & 2 \\
Haemangioma & 7 \\
Focal fatty infiltration & 9 \\
Focal nodular hyperplasia & 2 \\
Resolving hepatitis & 2 \\
Hypertrophic nodule & 1 \\
Total & 32 \\
\hline
\end{tabular}

patients with focal liver lesions it is useful to differentiate a hepatocellular carcinoma from metastases and to make a determination of the primary site of the tumour. In our study a correct diagnosis was made by histological examination in $93 \%$ of the cases; this overall accuracy is comparable with the results of cytological examination ${ }^{1-5}$ but in contrast with cytology using histological examination there were no false positives. Histological examination enables not only the differentiation between benign and malignant focal lesions but also gives a more precise tumour description " and makes it possible to determine the primary site of the tumour. In 15 patients with proved hepatocellular carcinoma we noted one advantage of the guided biopsy in that it enabled us to yield histological evidence of hepatocellular carcinoma. In this respect it is noteworthy that in six cases where laparoscopies and conventional biopsies were done, the diagnosis could not be verified by these procedures. By using real time scan in the routine and regular follow up of patients with chronic liver disease, either contour abnormalities or textural tissue echo abnormalities can often be detected.
Ultrasonography does not always permit a definite diagnosis of malignancy or benignity, especially when hepatoma develops on the underlying heterogeneous pattern of cirrhosis. Serum AFP measurement has been the most frequently used screening test for the diagnosis of hepatocellular carcinoma with a diagnostic yield of $15-65 \% .^{11-14}$ In our series of patients with proved hepatocellular carcinoma, raised serum concentrations of AFP were the first clue to diagnosis in only $46 \%$ of the cases. This comparatively low figure emphasises the need for ultrasonically guided biopsies in patients with cirrhosis and textural tissue echo abnormalities. In these cases histology ensures the differentiation between primary and secondary malignant liver lesions. Using cytology alone, the diagnosis of hepatocellular carcinoma is difficult - for example, in hepatitis or active cirrhosis cytology may show changes mimicking hepatocellular carcinoma. ${ }^{6}$ In the last analysis histological examination is the only method which can easily provide the diagnosis on which the choice of therapy depends.

Haemangiomas are the most frequent benign tumours of the liver and are present in $7 \%$ of the population. ${ }^{15}$ Characteristically in haemangiomas aspiration was followed by an immediate spurt of blood and no specimen could subsequently be obtained in most of these cases. Whenever such spurts of blood occurred the presence of haemangioma was suspected, a diagnostic advantage in that confirmation could easily be made by means of an Angio-computed tomography. ${ }^{16}$ On ultrasound examination fat in the liver produces increased echogenity. Focal fat is thus seen as an area of increased echogenity which makes it difficult for the clinician to differentiate between fat and malignant lesions. ${ }^{17}$ In contrast, histologic examination of the biopsies clearly showed an increased fatty infiltration of the liver and no malignant cells. In individual cases the interpretation of the histologic examination can also be difficult, especially in patients with known malignant disease. In these cases a sampling error has to be considered. In two patients guided fine needle biopsies were therefore repeated, but in both cases the same histologic result was revealed. The comparison of fine needle biopsies from the focal lesion and from the normal appearing liver might provide one way for a clearer diagnosis of focal fatty liver.

A theoretical objection to fine needle biopsy is the risk of causing widespread tumour cell dissemination. The risk of tumour spread, however, is insignificant when using the fine needle technique. ${ }^{9}$ Although the fine needle biopsy is considered safe it is nonetheless imperative that bleeding be considered. In one of our patients intra-abdominal bleeding did indeed occur. Generally speaking it can be said that the 
complication rate of fine needle biopsy is low, ${ }^{18}$ and even the possible angiomatous nature of hepatic lesions should not be considered an absolute contradiction to biopsy. ${ }^{19}$

\section{References}

1 Otto R, Wellauer J. Erfahrungen mit der ultraschallgezielten Feinnadelpunktion unter permanenter Sichtkontrolle. Roefo 1980; 133: 385-8.

2 Nosher JL, Plafker J. Fine-needle aspiration biopsy of the liver with ultrasound guidance. Radiology 1980; 136: 177-80.

3 Rosenblatt R, Kutcher R, Moussouris HF, Schrieber K, Koss LG, Sonographically guided fine-needle aspiration of liver lesions. JAMA 1982; 248: 1639-41.

4 Schwerk WB, Schmitz-Moormann P. Ultrasonically guided fine-needle biopsies in neoplastic liver disease. Cancer 1981; 48: 1469-77.

5 Ho CS, McLoughlin MJ, Tao LC, Blendis L, Evans WK. Guided percutaneous fine-needle aspiration biopsy of the liver. Cancer 1981; 47: 1781-5.

6 Johansen P, Svendsen KN. Scan-guided fine-needle aspiration biopsy in malignant hepatic disease. Acta Cytol 1978; 22: 292-6.

7 Schmitz-Moormann P, Schwerk W. Lebermetastasen. Zyto-histologische Untersuchungen. Pathologe 1984; 5: 260-1.

8 Atterbury CE, Enriquez RE, Desutonagy GI, Conn HO. Comparison of histologic and cytologic diagnosis of liver biopsies in hepatic cancer. Gastroenterology 1979; 76: $1352-7$.

9 Tao LC, Pearson FG, Delarue NC, Langer B, Sanders
DE. Percutaneous fine-needle aspiration biopsy. Cancer 1980; 45: 1480-5.

10 Tatsuta M, Yamamoto R, Kasugai H, et al. Cytohistologic diagnosis of neoplasms of the liver by ultrasonically guided fine-needle aspiration biopsy. Cancer 1984; 54: 1682-6.

11 Soreide O, Czerniak A, Blumgart LH. Large hepatocellular cancers: hepatic resection or liver transplantation? Br Med J 1985; 291: 853-7.

12 Shinagawa T, Ohto M, Kimura K, Tsunetomi S, Morita M. Diagnosis and clinical features of small hepatocellular carcinoma with emphasis on the utility of realtime ultrasonography. Gastroenterology 1984; 86: 495-502.

13 Sheu JC, Sung JL, Chen DS, et al. Early detection of hepatocellular carcinoma by real time ultrasonography. Cancer 1985; 56: 660-6.

14 Iwamura K. Hepatocellular carcinoma. In: Csomos G, Thaler H, eds. Clinical hepatology. Berlin: SpringerVerlag, 1983: 354-73.

15 Taylor KJW, Richmann TS. Diseases of the liver. Semin Roentgenol 1983; 18: 94-101.

16 Itai Y, Furin S, Araki T. Computed tomography of cavernous hemangioma of the liver. Radiology 1980; 137: 149-55.

17 Clain JE, Stephens DH, Charboneau JW. Ultrasonography and computed tomography in focal fatty liver. Gastroenterology 1984; 87: 948-52.

18 Livraghi T, Damascelli B, Lombardi C, Spagnoli I. Risk in fine-needle abdominal biopsy. $J$ Clin Ultrasound 1983; 11 : 77-80.

19 Solbiati L, Livraghi T, De Pra L, Ierace T, Masciadri N, Ravetto C. Fine-needle biopsy of hepatic hemangioma with sonographic guidance. AJR 1985; 144: 471-4. 